\title{
Incidence and survival rates of ovarian cancer in low-income women in Sudan
}

\author{
DAFALLA O. ABUIDRIS ${ }^{1}$, HSIN-YI WENG ${ }^{2}$, AHMED M. ELHAJ ${ }^{1}$, ELGAYLANI ABDALLAH ELTAYEB ${ }^{1}$, \\ MOHAMED ELSANOUSI ${ }^{4}$, REHAB S. IBNOOF ${ }^{4}$ and SULMA I. MOHAMMED ${ }^{2,3}$ \\ ${ }^{1}$ Oncology Department, National Cancer Institute, University of Gezira, Wad Madani, Sudan; ${ }^{2}$ Department \\ of Comparative Pathobiology, Purdue University; ${ }^{3}$ College of Veterinary Medicine, Purdue University Center for Cancer \\ Research, West Lafayette, IN, USA; ${ }^{4}$ Wad Medani Obstetrics and Gynecology Hospital, Wad Madani, Sudan
}

Received March 21, 2016; Accepted October 10, 2016

DOI: $10.3892 /$ mco.2016.1068

\begin{abstract}
Ovarian cancer is the second most common gynecological cancer worldwide. Little is known about the disease in Sudan. The objective of the present study was to evaluate the incidence rate, age and stage at diagnosis, and median survival time of patients presenting at the National Cancer Institute-University of Gezira (NCI-UG), Sudan. Data were collected in a prospective study of women with ovarian cancer over a period of eleven years of follow-up (between 2000 and 2011). Descriptive statistics were used to summarize the distribution of the demographics of the sample. The direct method was used to compute the age-standardized rate (ASR) using data from the 1966 and 2000 World Standard Populations (WSPs). The Kaplan-Meier method was used to estimate survival functions and the median survival time. Log-rank tests were used to statistically compare between the survival functions. There were steady increases in ovarian cancer incidence rates between 2000 and 2009, with a slight decline noted in 2010 and 2011. The patients' age range was 9-90. The age-specific incidence rate increased greatly in women aged 55 years or older. The majority of the patients had stage III or IV disease. The annual ASR using WSPs 1966 and 2000 as standard populations were 3.3 and 3.7 per 100,000 women, respectively. The median survival time was 31 months $(95 \%$ confidence interval, 19-43). The 5-year cumulative survival rate was $38 \%$. In Sudan, ovarian cancer affects postmenopausal women, akin to what is reported in the developed world with high incidence rates. Presenting with advanced stage disease is the predominant factor that results in a short survival time for women.
\end{abstract}

Correspondence to: Professor Sulma I. Mohammed, Department of Comparative Pathobiology, Purdue University, 725 Harrison Street, 1320 Lynn Hall, West Lafayette, IN 47907, USA

E-mail: mohammes@purdue.edu

Key words: ovarian cancer, papillary serrous cystadenocarcinoma, Africa, Sudan, Gezira State, survival, age-standardized rate

\section{Introduction}

Ovarian cancer is the seventh most common cancer, and it is the most common cause of mortality from gynecological cancers worldwide, with 238,619 incident cases in 2012 according to Globocan (http://globocan.iarc.fr/Default.aspx) (1).In developing countries, it is ranked the second most common gynecological cancer, and constitutes the fourth most common of all cancers in women, with 17,755 incident cases in 2012. Essentially, the highest incidence rates of ovarian cancer are found in the developed countries. Northern Europe has the highest incidence rate (13.3 per 100,000 person-years), followed by Western Europe (11.3 per 100,000 person-years) and Northern America (10.7 per 100,000 person-years), whereas North Africa has the lowest incidence rate (2.6 per 100,000 person-years) (2).

The incidence rate of ovarian cancer in the entire Sudan has yet to be identified; however, in a hospital-based data set from the National Cancer Institute, Gezira University, Central Sudan and Radiation Isotopes Center in Khartoum, collected between 2000 and 2006, ovarian cancer accounted for $6.8 \%$ (949) of all recorded cancers $(n=226,652)$, and it was ranked the sixth most common cancer for both genders (3). Additionally, in a more recent data set (2009-2010) from the National Cancer Registry for Khartoum State alone, ovarian cancer was the fourth most common cancer in women, with an estimated incidence rate of 188 per 100,000 population, a gender-specific rate of 8.0 per 100,000 population, and an age-standardized rate (ASR) of 7.0 per 100,000 population (4). Furthermore, neither the morality rate for ovarian cancer nor the survival rate in Sudan has previously been described due to a lack of the availability of death certificates, the majority of patients presenting with advanced stage disease were not thoroughly investigated or treated symptomatically.

Globally, a lack of reliable screening modalities has restricted the opportunities for early diagnosis and cancer detection, leading to a significant proportion of women worldwide presenting at an advanced stage of the disease. Due to this late presentation, available treatments are ineffective, and the majority of patients relapse following treatment-induced regression (5). Furthermore, there is substantial geographic variation in the incidence of ovarian cancer and mortality, with higher incidence observed in developed countries $(9.4$ per 
100,000 women) compared with women living in the developing world (5.0 per 100,000 women) (6).

The present study aimed to describe the time trends of the incidence rate of ovarian cancer in women diagnosed in Central Sudan between the years 2000 and 2011, as well as to investigate the age at diagnosis, histological type, stage, management and survival pattern of women with ovarian cancer presenting at the National Cancer Institute, Gezira University (NCI-UG), Sudan.

\section{Materials and methods}

The present study reports on patients with ovarian cancer who received treatment at NCI-UG, Wad Madani, Gezira State, Sudan, between January 2000 and December 2011. The NCI-UG offers gynecologic-oncological services to residents of Gezira State, as well as other neighboring states. All cases were referred to the NCI-UG, as it the only cancer center in the region to offer cancer treatment, as mentioned above. The Gezira, or Al Jazirah, State is located in central Sudan, south of the capital city, Khartoum. The present study was conducted with the approval of the NCI-UG's and Purdue University's Institutional Review Boards.

During the study period, Sudan was recognized as the largest country in the African continent, with a total area of 96,710 square miles, making the country slightly larger than one-quarter the size of the USA. Sudan is divided into 26 states and districts, with differing population densities. The states' Ministries of Health, Armed Forces, Universities, Police and private sector collectively, in an uncoordinated manner, provide health services to the people of Sudan. The public sector health services in Sudan are organized at three levels: Primary, secondary and tertiary. The states' general hospitals are the referral centers for the entire state (see the Federal Ministry of Health's website: http://ghdx. healthdata.org/organizations/federal-ministry-health-sudan).

The Radiation and Isotope Center in Khartoum (RICK) and NCI-UG at Wad Madani, Gezira State are the only two specialized cancer centers providing chemotherapy and radiotherapy services for all 26 states. Treatment is offered free for cancer patients. After exhausting all medical attempts at treatment at the primary and secondary care facilities, as well as local healers, patients are referred to RICK or NCI-GU, depending on the proximity to the patient's residence.

For the present study, data on the age of the patient at diagnosis, tumor pathology, stage of disease, treatment given, as well as demographic information (occupation, tribal affiliation), patient residence (urban/rural) and area of residence, were retrieved from the NCI-UG Cancer registry. The NCI-UG Cancer registry was the first national cancer registry to be established in Sudan (in 2006) with the support of the International Agency for Research (IARC). It uses the CanReg format (www.iacr.com.fr/canreg5.htm). Data are collected actively, as well as passively, and are checked for accuracy prior to being entered in the computer. Patients with a diagnosis of ovarian cancer from all gynecological hospitals in Gezira State and the surrounding states are referred to the NCI-UG. Usually, the referred patients will have a pathology or cytology report. In rare cases, for patients who were not fit for surgery or who had a negative cytology report, a clinical diagnosis was used [clinical presentation or imaging, in addition to an assessment of the tumor biomarker, cancer antigen 125 (CA125)].
Tumors were classified according to the tumor-lymph node-metastasis (TNM) classification, which is based on size of the primary tumor and presence of metastatic regional lymph nodes and/or of distant metastases (7). Few data on grade were available (note that this was not a common service at these local facilities). Tumors were graded as poorly differentiated, moderately differentiated or well differentiated. Staging was based on the primary operative report, and was performed according to the systems adopted by the International Federation of Gynecology and Obstetrics (FIGO) (8). Histological types of tumors were coded according to the International Classification of Diseases for Oncology (ICD-O) (9). The specific subtypes of tumors were as follows: Carcinoma, not otherwise specified (NOS), clear cell, papillary serous cystadenocarcinoma (PSCAD), moderately differentiated papillary serous adenocarcinoma (MDPSAD), moderately differentiated mucinous adenocarcinoma (MDMAD), endometrioid carcinoma (END/ENDOM), sex cord tumors (SCT), germ cell tumors (GCT) and mucinous cystadenocarcinoma (MCAD).

Patients were treated with surgery, and preoperative and postoperative chemotherapy. Patients were followed every 2 months during the first year, every 3 months during the second year, 4 months during the third year, and subsequently 6 months thereafter. During follow-up, patients were evaluated according to their history and standard diagnostic investigations, if required.

Statistical analysis. Descriptive statistics were presented to summarize the distribution of the demographics of the study sample. Different incidence rates of ovarian cancer, including the annual incidence rate, the age-specific rate and the ASR, were derived (10). The direct method was used to compute ASR using different standard populations, including the 2008 Sudan population and the 1966 and 2000 World Standard Populations (WSPs). The Kaplan-Meier method was used to estimate survival functions and median survival time. Log-rank tests were used to statistically compare between the survival functions. IBM SPSS Statistics for Windows, version 20.0 (IBM SPSS, Armonk, NY, USA) was used to analyze the data. $\mathrm{P}<0.05$ was taken to indicate a statistically significant value.

\section{Results}

Demographic data. During the period between January 2000 and December 2011, 341 ovarian cancer cases were diagnosed at the NCI-UG Center. Table I shows the distribution of ovarian cancers based on patient characteristics. The ages of the women at diagnosis ranged between 9 and 90 years, and the median age was 50 (standard deviation, \pm 16 years). Of the 341 women diagnosed with ovarian cancer, 239/341 (70\%) were from the Gezira State, whereas the remaining $30 \%$ were from the surrounding states, with the majority from Sinnar. Patients with ovarian cancer belonged to 40 tribal affiliations, with the majority belonging to Jaaleen (59/341; 17\%), Kawahla $(38 / 341 ; 11 \%)$, Rhfaa $(32 / 341 ; 9 \%)$ and Shaiqiah $(22 / 341 ; 6 \%)$.

Tumor histopathology. Histological classification of ovarian tumors for the 341 patients diagnosed with ovarian cancer at the NCI-UG is shown in Fig. 1. Germ cell tumors predominantly 
affected younger women, aged $<50$ years old $(25 / 30 ; 83 \%)$, and predominantly stage 1 patients $(50 \%)$.

Tumor stage. According to the TNM staging criteria, the majority of patients (73\%) were diagnosed with late-stage (stage III and IV) cancer (Table I).

Incidence of ovarian cancer. An increasing trend in the incidence rate of ovarian cancer was observed between 2000 and 2009, after which time it started declining in 2010 and 2011 (Fig. 2). The annual incidence rate peaked at 3 per 100,000 women in 2009. The ASRs on a yearly basis are presented in Fig. 3. The cumulative ASRs between the years 2000 and 2011 were measured as 22, 27 and 30 per 100,000 women using the 2008 Sudan population, the 1966 WSP and the 2000 WSP as the standard population, respectively. Similarly, the ASR increased between 2000 and 2009, and started to decline in 2010 and 2011 (Fig. 3). The incidence rate of ovarian cancer increased greatly in women aged 55 years or older (Fig. 4).

Surgical and chemotherapy management. Treatment consisted of a primary operation, followed by chemotherapy and/or radiotherapy. The standard surgical procedure at the NCI-UG and Wad Medani Obstetrics and Gynecology Hospital included total abdominal hysterectomy, bilateral salpingo-ophrectomy and omentectomy, which was performed in $37 \%$ of the cases. Other surgical procedures carried out by gynecologists outside of Wad Madani included salpingo-ophrectomy, bilateral salpingo-ophrectomy and partial surgery (performed in 7, 0.6 and $18 \%$ of the patients, respectively). Patients who were not fit for an operation were diagnosed using fine-needle aspiration cytology (17\%) and or tru-cut biopsy (35\%) and tumor marker (CA125) evaluation. Cases in which an operation was not possible, due to advanced disease, metastatic disease or the patient being unsuited for an operation, received neoadjuvant chemotherapy prior to a re-evaluation for complete surgery. Patients who underwent partial surgery prior to visiting the NCI-UG received either complete surgery or neoadjuvant chemotherapy first, and subsequently interval debulking surgery, depending on the response to chemotherapy and the patient's fitness.

Chemotherapy, used as a neoadjuvant therapy or post-operatively as an adjuvant therapy, depends on the stage and grade of the cancer, as per international guidelines (11). All patients (with the exception of stage I patients) received adjuvant chemotherapy. For stage I patients, only those with a high-grade tumor and positive cytology received adjuvant chemotherapy. The chemotherapy administered was carboplatin-based (carboplatin/ cyclophosphamide), and patients who had a poor response, or who suffered a relapse shortly after the chemotherapy, received second-line taxol-base chemotherapy (taxol/carboplatin). However, the taxol supply was not sustainable, due to financial reasons and also availability, and therefore Taxol/carboplatin could only be used as a second-line treatment. Approximately $27 \%$ (92/341) of patients with serous cystadenocarcinoma, or cancer not otherwise specified, received preoperative chemotherapy and, of those, $48 \%$ (44/92) received postoperative chemotherapy. Approximately $73 \%$ (249/341) of the patients received no preoperative chemotherapy; of those, $10 \%$ had germ cell tumors, $50 \%$ had serous cystadenocarcinoma, and $16 \%$ had
Table I. Distribution of ovarian cancers based on patient characteristics.

\begin{tabular}{lc}
\hline Variable & Frequency $(\%)$ \\
\hline Age total & $340(100)$ \\
$<15$ & $3(1)$ \\
$15-15$ & $16(5)$ \\
$25-54$ & $166(49)$ \\
$55-64$ & $75(22)$ \\
$65+$ & $80(23)$ \\
Stage total & $341(100)$ \\
0 & $4(1.2)$ \\
I & $57(1.7)$ \\
II & $32(9.4)$ \\
III & $169(49.6)$ \\
IV & $79(23.2)$ \\
Age-specific rate (per 100,000) & \\
$<15$ & 0.42 \\
$15-24$ & 4.13 \\
$25-54$ & 27.21 \\
$55-64$ & 109.34 \\
$65+$ & 111.08 \\
Occupation & $340(100)$ \\
Child & $2(1)$ \\
Employee & $19(5.5)$ \\
Farmer and worker & $20(5.8)$ \\
Housewife & $278(82)$ \\
Residence & $340(100)$ \\
Rural & $262(77)$ \\
Urban & $78(23)$ \\
&
\end{tabular}

${ }^{\mathrm{a}}$ Differences in the sample sizes were due to missing values.

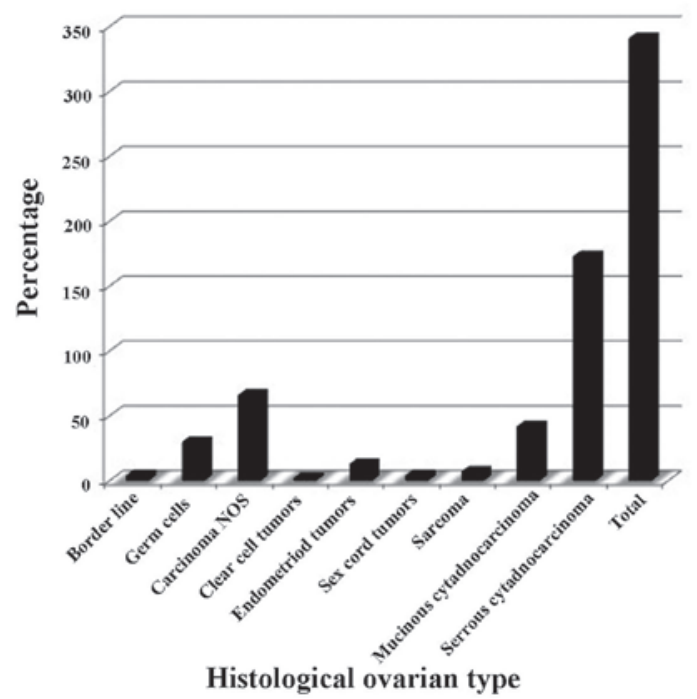

Figure 1. Histological distribution of ovarian cancer.

cancer not otherwise specified. Of those 249 patients, $16 \%$ received no postoperative chemotherapy. 


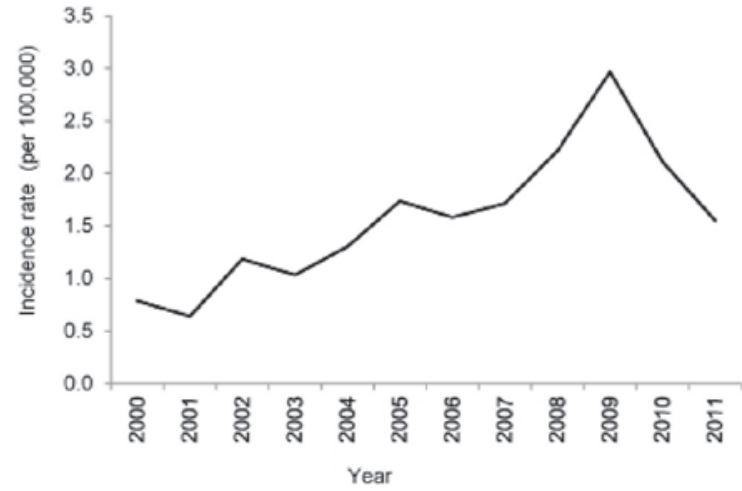

Figure 2. Annual incidence rates of ovarian cancer in Al Gezira State, Sudan, between the years 2000 and 2011.

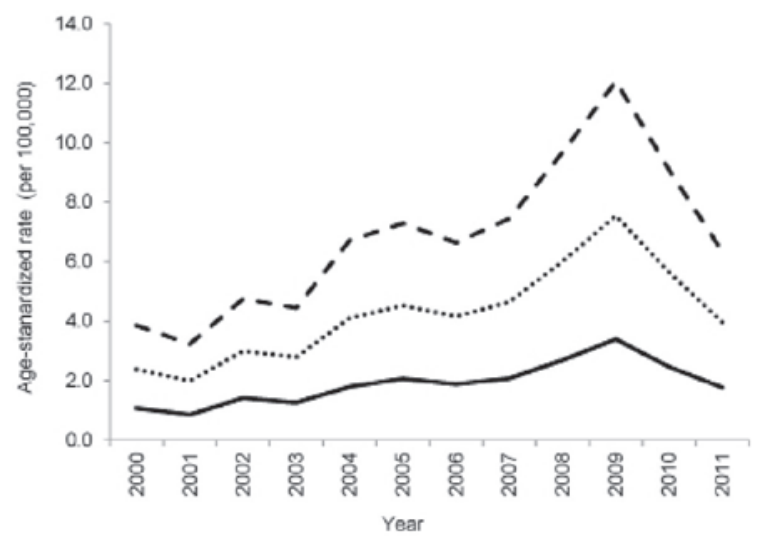

Figure 3. Rate of ovarian cancer between 2000 and 2011 using the 2000 WSP (dashed line), 1966 WSP (dotted line) and 2008 WSP (solid line) Sudan population. The $x$ and $y$-axes show the year and age-standardized incidence rates of ovarian cancer per 100,000 population, respectively. WSP, World Standard Population.

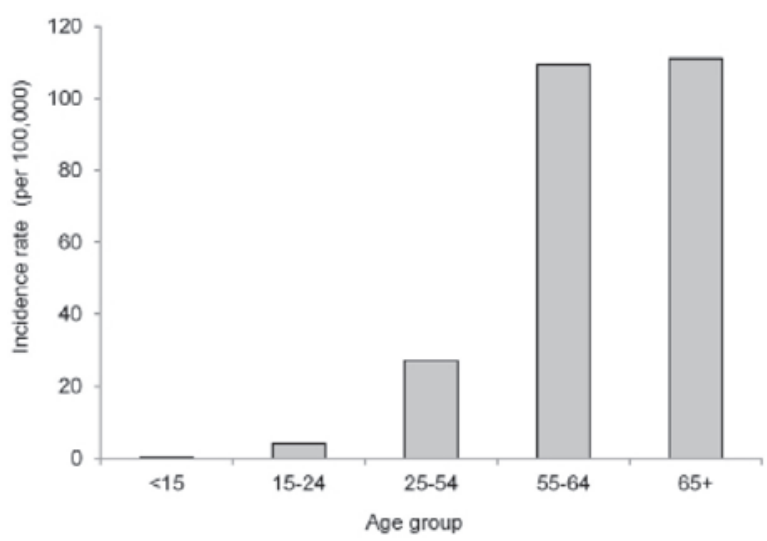

Figure 4. Age-specific rates of ovarian cancer in women in the Al Gezira State, Sudan, between the years 2000 and 2011.

Survival rate of patients with ovarian cancer. Of the 341 patients diagnosed at the NCI-UG Center between January 2000 and December 2011, 64/341 (19\%) were still alive, $39 \%$ were lost to follow up, and $42 \%$ had succumbed to mortality. The median survival time was 31 months $[95 \%$ confidence interval (CI), 19-43]. The 5-year cumulative survival rate of patients with ovarian cancer in Gezira State was $38 \%$ (95\% CI, 30-46\%). The cumulative survival rate was significantly different among the disease stages at the time of diagnosis $(\mathrm{P}<0.001)$. The median survival time in the stage III and IV patients was 23 (95\% CI, 18-28) and 15 (95\% CI, 3-27) months, respectively (Fig. 5A). Fig. 5B shows survival curves for patients living in urban or rural areas.

\section{Discussion}

The current study has presented data collected from women with ovarian cancer who visited the NCI-UG in Gezira State for diagnosis and treatment. Data on 341 patients were analyzed for the period between January 2000 and December 2011. Within this group of women, the median age at diagnosis was 50 years. Similarly young ages for the onset of cancer have been reported for women from other sub-Saharan African (12-14) and other (15) developing countries. However, it is different from that reported for developed countries, in which the median age at diagnosis is 61 years $(16,17)$. This may indicate that ovarian cancer affects young women in Africa. However, this observation of a younger age at diagnosis in African countries compared with other developed countries is likely to be due to the difference in population age distribution between the two: Africa has, by far, the youngest population of any of the continents (18). Given that age is the single substantive risk factor for the majority of cancers, including ovarian cancer, a younger population will have a lower overall incidence of ovarian cancer and a lower median age of onset, based simply on the demographics of the population. In the present study, the age-specific incidence rate, derived from a consideration of the population size of a particular age group, was determined to be highest in the group comprising women aged $\geq 55$ years, i.e. postmenopausal women (Fig. 4), which is consistent with findings worldwide.

The present study has demonstrated that the majority of patients with ovarian cancer presented with late stage disease, i.e. stage III and IV. This late presentation of ovarian cancer is also observed in other sub-Saharan African countries $(12,19)$. This finding could be explained by the 'silent' nature of the disease and its non-specific symptoms that hinder early diagnosis, in addition to a lack of cancer awareness and education, the influence of local healers and witchcraft, and so forth. Additionally, the majority of patients live in rural areas, where transportation and financial affordability may also be influencing factors. More than $90 \%$ of the tumors were epithelial in origin, with a higher prevalence in older women. Germ cell tumors constituted $9 \%$ of all tumors, and these were mostly predominant in younger women, i.e. $<40$ years old (range: 16-39), a similar presentation to what has been evidenced in the developed countries (20).

The present study revealed a gradual increase in the annual incidence rate of ovarian cancer between the years 2000 and 2009, and a subsequent decline in the years 2010 and 2011 in Gezira State (Fig. 2). The increase observed during the first nine years in the incidence rate may be attributed to improvements in diagnostics and treatment services, due to the establishment of the NCI-UG institution, which is the first population-based cancer registry in the country in Gezira State. On the other hand, the decline in incidence rates may be a result of errors made in the data collection, or it may reflect a true decrease in the ovarian cancer incident rate. Similar 

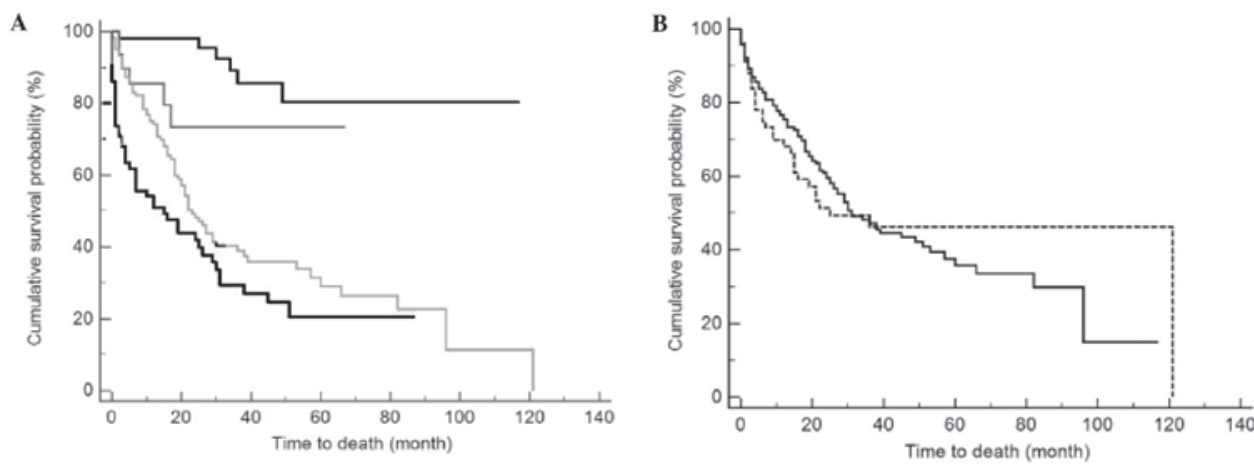

Figure 5. Kaplan-Meier plots showing the survival curves (A) according to the cancer stage (blue, stage 0 and I; green, stage II; orange, stage III; and black, stage IV) and (B) according to the location of the patient (rural area, continuous line; urban area, dashed line).

declines in the incidence rates of ovarian cancer have also been observed in Northern Europe, USA and China (12-24). An analysis of the data for 2012-2014 may assist in providing a more accurate picture regarding the trend in the incidence of ovarian cancer in Gezira State.

The world population age distribution has changed considerably, and therefore the World Health Organization (WHO) introduced, in the year 2000, a new WSP for the calculation of ASRs for future reports (25) to reflect more closely the age composition of the world's population, as projected over the subsequent 25 years. The goal of the new standard population is to minimize the disproportionate weighting of events in both the youngest and the oldest population groups (26). Using this new standard population, the computed ASRs of a number of cancer types, including ovarian cancer, are greatly increased (27). However, as the majority of previously published studies used the 1966 WSP to compute ASRs, they cannot be compared directly with the ASRs computed using the 2000 WSP. Therefore, in the present study, both 1966 and 2000 WSPs were used to calculate the ASR for ovarian cancer. The 2000-2011 cumulative ASRs using the 2008 Sudan population, 1966 WSP and 2000 WSP as standard population were determined to be 22, 27 and 30 per 100,000 women, respectively. The annual ASRs, using the 2008 Sudan population and WSPs 1966 and 2000 as standard populations, were 2.7, 3.3 and 3.7 per 100,000 women, respectively. Based on Globocan data (1) for 2008 using the 1966 WSP, the reported 3.3 per 100,000 ASR for Gezira State is lower compared with the ASR of all ovarian cancers reported worldwide (6.1 per 100,000), and with that of the entire Sudan (6.4), Africa (4.8), Sub-Saharan Africa (4.6), Eastern Africa (5.5) and Northern Africa (5.6). The highest ASRs reported for ovarian cancer were from the USA (8.1), all Europe (9.9) and Australia and New Zealand (7.6) (27). The increase in ovarian cancer in the developed world (USA, Europe and Australia and New Zealand) compared with sub-Saharan Africa may be accounted for by certain environmental factors and increased cancer awareness, combined with improved detection strategies.

At NCI-UG and the Wad Medani Obstetrics and Gynecology Hospital, ovarian cancers are managed by surgical debulking or cytoreduction of the tumor mass, followed by combination carboplatin-based chemotherapy, as per international guidelines. Treatments were administered according to the patients' age, disease stage, tumor histology and expected pregnancy. Surgical debulking or cytoreduction for advanced stage disease has long been shown to increase survival rates. In a meta-analysis of 53 studies of 6,885 patients with stage III and IV disease, it was reported that every $10 \%$ increase in surgical debulking is associated with a $5.5 \%$ increase in the median survival rate (28). The majority of patients $(45 \%)$ in the present study had surgical staging and debulking. Notably, of those patients, one-third were alive, one third had succumbed to mortality, and the remaining third were lost to follow up, preventing the formulation of any conclusions regarding the survival advantage of surgery in the current study.

Approximately $40 \%$ of the patients were lost during follow up in the present study. Similar percentages of patients lost to follow up were also observed in Ibadan (29) and Lagos, Nigeria (13). This issue is of concern specifically in the developing world in determining cancer survival rates, and has been discussed extensively by Sankaranarayanan et al (30). In the current study, Kaplan-Meier curves were used to determine survival rates and median survival times. Only the time up to the last visit of the patient was used in the computation of these survival measures. The loss to follow up may have resulted in a decrease in the reliability of the estimates, which would have been reflected in the $95 \% \mathrm{CI}$ estimates. Without further information on these patients following their last visit, it would not be possible to accurately predict any direct bias, if any, in the estimates. However, given that these patients did not follow through the recommended treatments, it would be expected that the survival measures estimated using the available data could have been overestimated.

In the present study, administration of neoadjuvant therapy did not alter the overall survival rate of the patients with ovarian cancer after adjusting for the disease stage at diagnosis $(\mathrm{P}=0.734)$. However, following stratification by disease stage, administration of adjuvant chemotherapy revealed effects on prolonging the survival rate in the stage III and IV patients $(\mathrm{P}=0.002$ and $\mathrm{P}<0.001$, respectively), but did not reveal any effects in the patients who were diagnosed at an earlier disease stage. The current study did not identify any additional survival benefits of neoadjuvant therapy compared with standard treatments of advanced ovarian cancer, as previously reported by others $(31,32)$.

A crossover of the survival functions at $\sim 40$ months was observed while comparing between the patients from rural 
and urban areas (Fig. 5B). Furthermore, based on a stratified analysis, no marked difference in the survival rate was identified between patients from rural (median survival time, 19 months) and urban (median survival time, 15 months) areas during the 40 months following the diagnosis $(\mathrm{P}=0.267)$. However, after this time point, the patients from urban areas (median survival time, 121 months) survived longer compared with the patients from rural areas (median survival time, 96 months), although the difference was not significant $(\mathrm{P}=0.062)$. The 5 -year cumulative survival rate of patients with ovarian cancer in Gezira State (38\%) was an improvement compared with those reported for different parts of Nigeria (29-33). However, the 5-year survival rate was lower compared with the United States, which may be explained by a lack of awareness and timely access to health care.

In addition, the current study has demonstrated that the FIGO stage was an independent prognostic factor for invasive ovarian cancer. The 5-year survival rate was greatly improved for early stage cancer compared with the advanced disease.

One limitation of the present study was the possibility of various errors in data collection and reporting, which may have occurred, particularly with data collected prior to the establishment of the cancer registry at NCI-UG. Another possible limitation of this study was that it did not include patients who may have left NCI-UG and were subsequently treated at the Khartoum Radiation and Isotope Center.

In Gezira State, the incidence rate for ovarian cancer exhibited an increasing trend during the period between 2000 and 2009. It is more common in postmenopausal women, which is in agreement with reports from the developed world (6). Women presented at an advanced stage of the disease, which resulted in short survival times. To the best of our knowledge, this is the first study to determine the survival time for women with ovarian cancer in the entire Sudan, and it should serve as a basis from which to monitor the effectiveness of ovarian cancer management in Sudan in the future.

\section{Acknowledgements}

The National Cancer Institute, University of Gezira supported the Study.

\section{References}

1. Ferlay J, Ervik M, Dikshit R, Eser S, Mathers C, Rebelo M, Parkin DM, Forman D and Bray F: Cancer incidence and mortality worldwide: IARC CancerBase No. 11.

2. Parkin DM, Bray F, Ferlay J and Pisani P: Global cancer statistics, 2002. CA Cancer J Clin 55: 74-108, 2005.

3. Mohammed ME, Hassan AM, Abdelhadi HA, Elsadig MG, Adam DM, Elmamoun K, Hamid R, Elias H, Abdallah M, Abdelkarim Z, Elwali NE and Mohammed SI: Burden and pattern of cancer in the Sudan, 2000-2006. Br J Med Med Res: 4: $1231-1243,2014$.

4. Saeed IE, Weng HY, Mohamed KH and Mohammed SI: Cancer incidence in Khartoum, Sudan: First results from the cancer registry, 2009-2010. Cancer Med 3: 1075-1084, 2014.

5. Holschneider $\mathrm{CH}$ and Berek JS: Ovarian cancer: Epidemiology, biology, and prognostic factors. Semin Surg Oncol 19: 3-10, 2000.

6. Jemal A, Bray F, Center MM, Ferlay J, Ward E and Forman D: Global cancer statistics. CA Cancer J Clin 61: 69-90, 2011.

7. Greene FL, Page DL, Fleming ID, Fritz A, Balch CM, Haller DG and Morrow M (eds): American Joint Committee on Cancer: AJCC Cancer Staging Manual. 6th edition. Springer, New York, NY, pp157-164, 2002.

8. Shepherd JH: Revised FIGO staging for gynaecological cancer. Br J Obstet Gynaecol 96: 889-892, 1989.
9. Fritz AG: International classification of diseases for oncology: ICD-O. World Health Organization, 2000.

10. Naing NN: Easy way to learn standardization: direct and indirect methods. Malays J Med Sci 7: 10-5, 2000.

11. Morgan RJJ, Alvarez RD, Armstrong DK, et al: NCCN clinical practice guidelines in oncology: epithelial ovarian cancer. J Natl Compr Cancer Netw 9: 82-113, 2011.

12. Vanderpuye V and Yarney J: Ovarian cancer: An analysis of forty-four patients at the national radiotherapy centre, Accra-Ghana. West Afr J Med 26: 93-96, 2007.

13. Rabiu KA, Akinola OI, Adewunmi AA, Fabamwo AO, Adedeji MO and Popoola AO: Delays in presentation and management of ovarian cancer in Lagos, Nigeria. J Obstet Gynaecol 33: 305-308, 2013.

14. Doh AS and Shasha W: A clinico-pathological study of ovarian tumors in Yaounde-Cameroun. West Afr J Med 13: 196-199, 1994.

15. Basu P, De P, Mandal S, Ray K and Biswas J: Study of 'patterns of care' of ovarian cancer patients in a specialized cancer institute in Kolkata, eastern India. Indian J Cancer 46: 28-33, 2009.

16. Quirk JT and Natarajan N: Ovarian cancer incidence in the United States, 1992-1999. Gynecol Oncol 97: 519-523, 2005.

17. van Altena AM, Karim-Kos HE, de Vries E, Kruitwagen RF, Massuger LF and Kiemeney LA: Trends in therapy and survival of advanced stage epithelial ovarian cancer patients in the Netherlands. Gynecol Oncol 125: 649-654, 2012.

18. Harford JB: Breast-cancer early detection in low-income and middle-income countries: Do what you can versus one size fits all. Lancet Oncol 12: 306-312, 2011.

19. Gharoro EP and Eirewele O: Cancer of the ovary at the university of benin teaching hospital: A 10-year review, 1992-2001. Afr J Med Med Sci 35: 143-147, 2006.

20. Sankaranarayanan R and Ferlay J: Worldwide burden of gynaecological cancer: The size of the problem. Best Pract Res Clin Obstet Gynaecol 20: 207-225, 2006.

21. Bray F, Loos AH, Tognazzo S and La Vecchia C: Ovarian cancer in Europe: Cross-sectional trends in incidence and mortality in 28 countries, 1953-2000. Int J Cancer 113: 977-990, 2005.

22. Morris CR, Rodriguez AO, Epstein J and Cress RD: Declining trends of epithelial ovarian cancer in California. Gynecol Oncol 108: 207-213, 2008.

23. Lowe KA, Chia VM, Taylor A, O'Malley C, Kelsh M, Mohamed M, Mowat FS and Goff B: An international assessment of ovarian cancer incidence and mortality. Gynecol Oncol 130: 107-114, 2013.

24. Wong KH, Mang OW, Au KH and Law SC: Incidence, mortality, and survival trends of ovarian cancer in Hong Kong, 1997 to 2006: A population-based study. Hong Kong Med J 18: 466-474, 2012.

25. Ahmad OBB-PC, Lopez AD, Murray CJL, Lozano R and Inoue M: Age standardization of rates: A new WHO standard. Global programme on evidence for health policy discussion paper Series: no. 31. Geneva, 2000.

26. Bray F, Guilloux A, Sankila R and Parkin DM: Practical implications of imposing a new world standard population. Cancer Causes Control 13: 175-182, 2002.

27. Bray F, Ren JS, Masuyer E and Ferlay J: Global estimates of cancer prevalence for 27 sites in the adult population in 2008 . Int J Cancer 132: 1133-1145, 2013.

28. Bristow RE, Tomacruz RS, Armstrong DK, Trimble EL and Montz F: Survival effect of maximal cytoreductive surgery for advanced ovarian carcinoma during the platinum era: A meta-analysis. J Clin Oncol 20: 1248-1259, 2002.

29. Adekanbi AA, Olayemi O, Okolo CA, Fawole AO, Odukogbe AT and Okani CO: Survival of ovarian cancer patients in Ibadan: Clinical and pathological factors. J Obstet Gynaecol 34: 57-59, 2014.

30. Sankaranarayanan R, Black RJ, Swaminathan R and Parkin DM: An overview of cancer survival in developing countries. IARC Sci Publ: 135-157, 1998.

31. Tangjitgamol S, Manusirivithaya S, Laopaiboon M, Lumbiganon $\mathrm{P}$ and Bryant A: Interval debulking surgery for advanced epithelial ovarian cancer. Cochrane Database Syst Rev: CD006014, 2016.

32. Bristow RE, Eisenhauer EL, Santillan A and Chi DS: Delaying the primary surgical effort for advanced ovarian cancer: A systematic review of neoadjuvant chemotherapy and interval cytoreduction. Gynecol Oncol 104: 480-490, 2007.

33. Iyoke C, Ugwu G, Ezugwu E, Onah N, Ugwu O and Okafor O: Incidence, pattern and management of ovarian cancer at a tertiary medical center in Enugu, South East Nigeria. Ann Med Health Sci Res 3: 417-421, 2013. 